\title{
Invasive pulmonary aspergillosis in a HIV-infected patient with metastatic, non-small cell lung cancer
}

\author{
Joanna Puła, Agnieszka Bednarska, Marcin Paciorek
}

Hospital of Infectious Diseases, Department of Infectious Diseases for Adults, Medical University of Warsaw, Poland

\begin{abstract}
Aspergillus species remains an important cause of morbidity and mortality in immunosuppressed patients. The following case report presents a 56-year-old HIV-infected patient on successful combined antiretroviral therapy (cART), who was diagnosed with non-small cell lung cancer in November 2015 and invasive aspergillosis in January 2016. At that time, we noticed a sudden decrease of his CD4+ count from 758 cells/ $\mu$ in October 2015 to 48 cells/ $\mu$ in January 2016. It was probably not a result of HIV infection, because viral load had been undetectable, and the CD4+ count had remained $>500$ cells/ $\mu$ l for many years. It is more likely that the patient's severe lymphopenia was caused by metastatic cancer and the treatment (radiotherapy) that had been applied. There is data showing that a significant decrease of the CD4+ count is a frequent side effect in patients following treatment with radiation and chemotherapy for newly diagnosed solid tumors, regardless of histopathology and the type of chemotherapy. Both advanced AIDS and immune deficiencies connected with solid organ tumors are risk factors for invasive pulmonary aspergillosis and as the case described illustrates, these two medical conditions are often connected with severe lymphopenia. The question that arises is whether severe lymphopenia constitutes an independent risk factor for invasive pulmonary aspergillosis.
\end{abstract}

HIV AIDS Rev 2017; 16, 3: 195-197 DOI: https://doi.org/10.5114/hivar.2017.70719

Key words: invasive pulmonary aspergillosis, HIV infection, immunodeficiency, lung cancer, metastatic cancer, lymphopenia.

\section{Case report}

In October 2015, a 56-year-old HIV-infected man presented with a focal change in the right lung. He had smoked for 20 years.

His HIV infection was diagnosed in 2002. From March 2004 to January 2010, he received: Epivir, Ziagen, and Kaletra. From the end of January till April 2011, patient's therapy was interrupted. Treatment with Ziagen, Retrovir, Prezista, and Norvir was established, and continued until the end of his life. Table 1 presents the viral and an immunological response to highly active antiretroviral therapy (HAART).
The patient had a chest X-ray in October 2015 revealing a nodular change next to the trachea on the right. Computed tomography (CT) of the chest showed a tumor of $60 \mathrm{x} 53 \mathrm{x}$ $56 \mathrm{~mm}$ in segments 1 and 2 of the right lung adhering to the mediastinal pleura, trachea, and bronchus. Furthermore, a nodule of $15 \times 20 \mathrm{~mm}$ was found in segment 1 of the left lung. Tuberculosis was excluded (bronchoalveolar lavage tests, a microscopic examination, and the polymerase chain reaction [PCR] test of Mycobacterium tuberculosis were all negative), and the patient was transferred to the Institute of Tuberculosis and Lung Diseases in Warsaw where he
Address for correspondence: Joanna Puła, MD,

Hospital of Infectious Diseases, Department of Infectious Diseases

for Adults, Medical University of Warsaw,

37 Wolska St., 01-201 Warsaw, Poland,

e-mail: joannapula@wp.pl
Article history:

Received: 31.05.2017

Received in revised form: 23.06.2017

Accepted: 29.07.2017

Available online: 07.10.2017
International Journal of HIV-Related Problems

HIV \& AIDS

R e vi e w 
Table 1. Viral and an immunological response to highly active antiretroviral therapy

\begin{tabular}{|c|c|c|c|c|c|c|c|c|c|c|c|c|c|}
\hline & 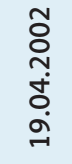 & 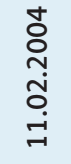 & 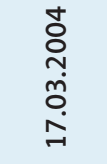 & 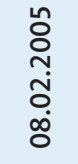 & 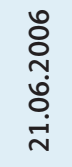 & 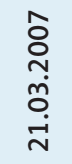 & 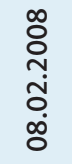 & $\begin{array}{l}\text { ᄋे } \\
\stackrel{i}{+} \\
\stackrel{-}{-1} \\
\stackrel{+}{+}\end{array}$ & 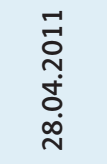 & 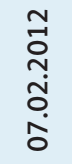 & $\begin{array}{l}m \\
\text { ō } \\
\text { ọ } \\
\text { ọ } \\
\stackrel{-}{-}\end{array}$ & 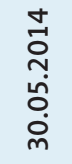 & 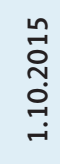 \\
\hline Viremia; HIV copies/ml & & & 69,400 & $<50$ & ud & 62 & $<40$ & 5,649 & 31,860 & ud & ud & ud & ud \\
\hline CD4+; Cells/ $\mu \mathrm{l}$ & 529 & 224 & & 548 & 622 & 469 & 581 & 526 & 373 & 570 & 571 & 589 & 758 \\
\hline CD4+/CD8+ & 0.41 & 0.22 & & 0.50 & 0.53 & 0.52 & 0.57 & 0.36 & 0.24 & 0.32 & 0.32 & 0.40 & 0.43 \\
\hline
\end{tabular}

underwent bronchoscopy. Histopathological examination of a tumor section revealed non-small cell neuroendocrine lung cancer. Diagnosis and treatment continued in the Centre of Oncology in Warsaw.

Computed tomography of the head showed 3 metastases in the brain: in the right frontal lobe, in the right temporal lobe, and in the left occipital lobe. Palliative radiotherapy of the chest and head was applied in December 2015.

At the beginning of January 2016, the patient lost consciousness in the street and was taken to Orłowski's Hospital in Warsaw, and then to the Hospital of Infectious Diseases. On admission, he was in an average general state, with good verbal-logical contact, whitish fungal coating in the mouth, and auscultation crackles at the bottom of his lungs, as well as a decrease of the alveoli murmur on the left, saturation $90 \%$ without oxygen therapy, tachycardia $102 / \mathrm{min}$, and BP 95/70. Inflammatory parameters were high in blood tests CRP $1120 \mathrm{mg} / \mathrm{l}$. A chest X-ray revealed parenchymal densities at the base of the left lung, encysted liquid in the left cavity pleura. The results of aerobic and anaerobic blood cultures were negative. Empiric therapy of ceftriaxone and doxycyline were applied, with fluconazole due to oral candidiasis. We used reactive oxygen therapy gaining an improvement of the patient's clinical state, i.e., a relief of his shortness of breath, an increase of saturation to $97 \%$ without oxygen therapy, and an improvement of blood tests as well as a decrease of CRP to $11 \mathrm{mg} / \mathrm{l}$. A control chest X-ray showed a cavity with the characteristics of tissue breakdown and a liquid with a morphology, which was non typical for a cancer. Chest CT revealed a lot of small solid changes in both lungs, a cavity next to a hilum of the right lung and a bigger one (diameter $14 \mathrm{~cm}$ ) in the lower lobe of the left lung connected with the pleura, some liquid and air blisters in the left cavity pleura. In the next chest X-ray, we saw a left-sided pneumothorax and a partly collapsed left lung. A thoracic surgeon was consulted who decided to do bronchoscopy. The bronchoalveolar lavage test results were as follows: the microscopic examination and PCR test of Mycobacterium tuberculosis were negative, while a culture positive for Staphylococcus saprophyticus MRSS, Aspergillus species was found. Pleural drainage was indispensable. The microbiological examination of the pleural fluid was positive and proved Aspergillus species infection. Treatment with voriconazole and vancomycin was introduced. In the following days, there was a decrease of fluid drainage, an improvement in imaging tests but a fistula between the cavity and pleura remained, therefore Heimlich's valve was connected to the drainage set. At the same time, we observed a low level of lymphocytes in lab tests, so the immunological profile was checked and it detected severe lymphocytopenia: CD4+ 48 cells/ $\mu$ l, CD4+/CD8+ 0.2. Viral load was undetectable. We expanded the antibiotic therapy adding azithromycin and trimethoprim/sulfamethoxazole as prophylaxis against opportunistic diseases.

The patient was released from hospital on 4 February 2016 and directed to his home hospice. Treatment with voriconazole, as well as the above-mentioned prophylaxis were continued. He died on 1 March 2016 at home.

\section{Discussion}

Invasive aspergillosis remains a major cause of morbidity and mortality in immunosuppressed patients. The most important risk factor is neutropenia, especially if the total neutrophil count decreases below 500 cells/ $\mu$ l. Moreover, the risk of invasive pulmonary aspergillosis (IPA) is highly correlated with the duration and degree of neutropenia. Other frequent risk factors are transplantation (the highest risk occurs in cases of lung transplantation and HSCT), prolonged (> 3 weeks), or high-dose corticosteroid therapy, hematological malignancy (the risk is higher in leukemia), chemotherapy, advanced AIDS, and chronic granulomatous disease [1]. Other infrequent risk factors are immune deficiencies connected with solid organ tumors, chronic obstructive pulmonary disease (COPD), or critically ill patients [1-3].

The diagnosis of IPA is still a challenge. Histopathological examination of lung tissue remains the gold standard. The presence of septate, branching hyphae invading lung tissue with a culture positive for Aspergillus allows us to diagnose and confirm a case of invasive pulmonary aspergillosis $[1,3]$. A new pulmonary infiltrate on the chest radiograph and a positive bronchoalveolar lavage fluid or sputum culture for Aspergillus species let us diagnose a probable case of IPA $[1,3]$; it requires considering the patient in the context of his immune status. In immunocompetent patients, the isolation of Aspergillus spp. usually means colonization. 
There was a study of 66 patients after hospitalization who had sputum cultures positive for Aspergillus spp. Among these, $92 \%$ suffered from colonization and only $4.5 \%$ from IPA [4], whereas isolation of Aspergillus in immunosuppressed people like in patients with leukemia, or in those who had undergone HSCT, has a positive predictive value of $80-90 \%$. On the other hand, negative cultures do not foreclose IPA. $70 \%$ of the patients with confirmed pulmonary aspergillosis had negative sputum samples [5].

To improve our diagnostic process, we can use imaging studies. A chest radiograph can reveal rounded densities, pleural-based infiltrates suggestive of pulmonary infarctions, and cavitations. Chest CT, especially with high-resolution images (HRCT) is a much better procedure that can show us well demarcated multiple nodules with or without the halo sign that is an effect of hemorrhage surrounding the pulmonary nodule. Progression of these changes can result in cavities or the air-crescent sign, which are images of necrotic lesions of inflammation [6]. None of the above changes are pathognomonic for aspergillosis.

In HIV-infected patients who have a good immunological and viral response to HAART, IPA is not a major problem. Most cases of IPA were connected with lymphocytes CD4+ count $<50$ cells/ $\mu$ l. Moreover, half of these patients were treated with steroids or had neutropenia. The average survival time among these people is 3 months $[3,7,8]$.

Since in the case described here there was no histopathological examination, what we discussed above is an example of a probable case of invasive pulmonary aspergillosis. However, a positive bronchoalveolar lavage (BAL) culture and pleura fluid culture, changes in chest CT (nodules, cavities), improvement after antifungal treatment, and risk factors suggest that it was the right diagnosis.

When we have studied the course of the illness of our patient, we noticed a sudden decrease of CD4+ count from 758 cells/ $\mu$ in October 2015 to 48 cells/ $\mu$ l in January 2016. We can suspect that paradoxically it was not a result of HIV infection, because a viral load was undetectable and the CD4+ count remained $>500$ cells/ $\mu$ l for many years. It is controversial whether we can say that the HIV infection evolved into AIDS in this patient. On the one hand, we have observed the patient's severe immunodeficiency, and pulmonary aspergillosis is a disease indicator of this syndrome. On the other hand, both of these factors resulted from metastatic cancer and the treatment (radiotherapy) that had been applied, not HIV infection and the syndrome defined by the AIDS acronym assigned to the infection of this particular virus. Probably HIV infection made the patient predisposed to lymphopenia due to the degradation of his lymph nodes. The virus replicates mainly in lymph nodes, where a continuous inflammation leads to fibrosis. This process in turn entails structural and functional disorders of lymph nodes that limit reconstruction of the immune system, even after the introduction of effective HAART [9]. Despite this, we cannot say that in the case HIV infection developed into AIDS, so we concluded that our patient had severe lymphopenia, not AIDS.
The following data present the survival of patients with severe lymphopenia following treatment with radiation and chemotherapy for newly diagnosed solid tumors: malignant glioma $(n=96)$, resected pancreatic cancer $(n=53)$, unresectable pancreatic cancer $(n=101)$, and non-small cell lung cancer $(n=47)$. The results show that two months after initiating chemoradiation, $43 \%$ of patients developed severe and persistent lymphopenia $(<500$ cells $/ \mu \mathrm{l})$, regardless of histopathology and the type of chemotherapy. Further lymphopenia was connected with shorter survival independently of cancer progression [10].

Regarding risk factors for invasive pulmonary aspergillosis, advanced AIDS and immune deficiencies are connected with solid organ tumor. Maybe these two risk factors have a common point, i.e., severe lymphopenia that could constitute an independent risk factor. The second question that arises is whether it would be justified to introduce prophylaxis against opportunistic infections after oncologic treatment.

\section{Conflict of interest}

The author's declared no potential conflicts of interest with respect to the research, authorship, and/or publication of this article.

\section{References}

1. Kousha M, Tadi R, Soubani AO. Pulmonary aspergillosis: a clinical review. Eur Respir Rev 2011; 20: 121, 156-174.

2. Petterson TF, Kirkpatrick WR, White M, et al. Invasive Aspergillosis. Disease Spectrum, Treatment Practices and Outcomes. Medicine 2000; 79: 250-260.

3. Lortholary O, Meyohas MC, Dupont B, et al. Invasive aspergillosis in patients with acquired immunodeficiency syndrome: report of 33 cases. French Cooperative Study Group on Aspergillosis in AIDS. Am J Med 1993; 95: 177-187.

4. Soubani AO, Khanchandani G, Ahmed HP. Clinical significance of lower respiratory tract aspergillus culture in elderly hospitalized patients. Eur J Clin Microbiol Infect Dis 2004; 23: 491-494.

5. Yu VL, Muder RR, Poorsattar A. Significance of isolation of Aspergillus from the respiratory tract in diagnosis of invasive pulmonary aspergillosis. Results from a three-year prospective study. Am J Med 1986; 81: 249-254.

6. Kuhlman JE, Fishman EK, Siegelman SS. Invasive pulmonary aspergillosis in acute leukemia: characteristic findings on CT, the CT halo sign, and the role of CT in early diagnosis. Radiology 1985; 157: 611-614.

7. Denning DW, Follansbee SE, Scolaro M, et al. Pulmonary aspergillosis in the acquired immunodeficiency syndrome. N Engl J Med 1991; 324: 654-662.

8. Mylonakis E, Barlam TF, Flanigan T, et al. Pulmonary aspergillosis and invasive disease in AIDS: review of 342 cases. Chest 1998; 114: 251-262.

9. Schacker TW, Nguyen PL, Martinez E, et al. Persistent abnormalities in lymphoid tissues of human immunodeficiency virus-infected patients successfully treated with highly active antiretroviral therapy. J Infect Dis 2002; 186: 1092-1097.

10. Grossman SA, Ellsworth S, Campian J, et al. survival in patients with severe lymphopenia following treatment with radiation and chemotherapy for newly diagnosed solid tumors. J Natl Compr Canc Netw 2015; 13: 1225-1231. 